\title{
The safety of early versus late ileostomy reversal after low anterior rectal resection: a retrospective study in 47 patients
}

\author{
lan Fukudome ${ }^{\text {* }}$, Hiromichi Maeda', Ken Okamoto², Hajime Kuroiwa3', Sachi Yamaguchi', Kazune Fujisawa', \\ Mai Shiga', Ken Dabanaka', Michiya Kobayashi' ${ }^{1}$, Tsutomu Namikawa ${ }^{1}$ and Kazuhiro Hanazaki ${ }^{1}$
}

\begin{abstract}
Background: This study aimed to clarify the safety of early closure in diverting ileostomy with lower anterior rectalcancer resection.

Methods: We retrospectively reviewed consecutive 47 patients who underwent diverting ileostomy with lower rectal-cancer resection between May 2009 and October 2017. The results of the stoma closure were compared between patients who underwent stoma closure within 90 days (early closure [EC] group) and those who underwent late closure (LC group; closure after 90 days). Because of the small sample size, the frequency of severe complications post closure was analyzed.

Results: Among 47 patients, 29 were in the EC group. Postoperative complications occurred in $48.3 \%$ (14/29) and $27.8 \%(5 / 18)$ of patients in the EC and LC groups, respectively. This difference was due to minor complications (Clavien-Dindo Classification I/II), such as superficial incisional surgical site infections $(n=5)$ in the EC group. The rate of severe complications (Clavien-Dindo Classification $\geq$ III) was similar between the groups (20.7\% vs. $16.7 \%, p=1$, Fisher's exact test).

Conclusions: No association was observed between the time of closure and development of major complications; however, there was an increased likelihood of minor complications after EC. This study provides a basis on which future treatment guidelines for early stoma closure may be developed without affecting patient quality of life.
\end{abstract}

Keywords: Stoma closure, Low anterior resection, Diverting ileostomy

\section{Background}

Anastomotic leakage is one of the most serious complications after lower anterior resection, with an occurrence rate of $3-28 \%$ based on a meta-analysis and literature review $[1,2]$. The preventive role of the transanal rectal drainage tube is controversial [3-5], and creation of a diverting ileostomy is reliable for the prevention of an anastomotic leakage $[6,7]$. Stoma closure is

\footnotetext{
* Correspondence: iamian0317@yahoo.co.jp

'Department of Surgery, Kochi Medical School, Kohasu, Oko-cho, 783-8505 Nankoku-city, Kochi, Japan

Full list of author information is available at the end of the article
}

generally scheduled 3-6 months after the initial surgery, when wound healing is uneventful $[8,9]$. However, a diverting ileostomy may cause major psychological and physical stress, leading to a decrease in the quality of life $[10,11]$; thus, patients often request an earlier reversal.

A few randomized clinical trials have elucidated the optimal timing for reversal of the diverting ileostomy $[12,13]$. Although the safety of very early stoma closure (within 2 weeks), compared to conventional stoma closure (after 3 months), was suggested, each of these trials has limitations. A recent trial comparing the safety of closing an ileostomy within $8-13$ days after rectal

(C) The Author(s). 2021 Open Access This article is licensed under a Creative Commons Attribution 4.0 International License, which permits use, sharing, adaptation, distribution and reproduction in any medium or format, as long as you give appropriate credit to the original author(s) and the source, provide a link to the Creative Commons licence, and indicate if changes were made. The images or other third party material in this article are included in the article's Creative Commons licence, unless indicated otherwise in a credit line to the material. If material is not included in the article's Creative Commons licence and your intended use is not permitted by statutory regulation or exceeds the permitted use, you will need to obtain permission directly from the copyright holder. To view a copy of this licence, visit http://creativecommons.org/licenses/by/4.0/ The Creative Commons Public Domain Dedication waiver (http://creativecommons.org/publicdomain/zero/1.0/) applies to the data made available in this article, unless otherwise stated in a credit line to the data. 
resection and 12 weeks after initial surgery demonstrated a similar rate of severe postoperative complications between the groups [12]. However, the inclusion of 127 of the 418 patients who were assessed for eligibility suggests that this study had strict patient selection criteria. Alves et al. reported that early stoma closure on day 8 after initial surgery was feasible, with reduced hospital stay, bowel obstruction, and medical complications [13]. However, the recruited patients were young, with a mean age of 58 years, and seemed to have clinical characteristics different from those of subjects in our country $[14,15]$ and institutes [16]. Although colorectal surgery has been proven to be safe in elderly patients [16], repeated surgical interventions within a short period of time must be carefully assessed in patients with poor general health.

Owing to the lack of enough evidence concerning early stoma closure, this retrospective study aimed to evaluate the safety of early closure (EC) $(<90$ days after operation) of diverting ileostomy created during lower anterior resection for rectal cancer by reviewing the clinical data of our patients.

\section{Methods}

\section{Patient groups}

The data of consecutive patients $(N=50)$ who underwent lower anterior resection for rectal tumors and diverting ileostomy at the Kochi Medical School Hospital in Japan between May 2009 and October 2017 were collected. After excluding two cases with a simultaneous hepatectomy and one case with failure to close the diverting ileostomy, we analyzed the data of 47 patients. Because stoma closure is often scheduled 3 months after the initial operation, we determined to divide the patients into 2 groups at 90 days. Those who underwent stoma closure within 3 months (within 90 days) were grouped into the EC group, while those who underwent stoma closure after 90 days or more were grouped into the late closure group (LC group). Data regarding complications developed within 1 year of stoma closure were collected. This study was approved by the Institutional Review Board of the Kochi Medical School (ERB-103, 996, 30-44), with an opt-out system in place for participant consent.

\section{Operation and management}

Lower anterior resection was performed with total mesorectal excision [17] or tumor-specific mesorectal excision and regional lymphadenectomy. Pelvic lateral lymph node dissection was not routinely performed in our institute. Instead, resection or chemo-radiation was performed for the swollen nodes detected by preoperative imaging tests. End-to-end anastomosis was performed using the double-stapling technique or trans- anal coloanal anastomosis. When anastomosis was performed at the level of the levator ani muscle, a diverting ileostomy was created. Water intake was usually allowed on the first postoperative day, and oral intake of solid food was initiated from the second postoperative day. Typically, we closed the diverting ileostomy within 3-6 months after the initial operation; however, no stipulated guideline currently exists regarding ileostomy closure. For the test of anastomosis, the Barium enema and CT scan before stoma colure was performed the most frequently, while endoscopy was rarely performed.

The procedure for ileostomy closure began with a skin incision made in a spindle-like fashion around the ileostomy. Closure of the intestinal orifice with $1-0$ silk sutures (1 - 0 Braided Silk, Akiyama-seisakusho. CO., Ltd, Japan) was then performed to maintain a clear operative field. Next, the ileum was circumferentially dissected and mobilized from the abdominal wall. After lifting the stoma out of the wound, a functional end-to-end anastomosis with an automatic suturing device (ETHICON Linear cutters, Johnson and Johnson MEDICAL DEVI CES COMPANIES, America) was performed. The peritoneum, posterior layer of the fascia, and anterior layer of the fascia were closed, separately. After washing the subcutaneous tissue with normal saline (OTSUKA NORMAL SALINE, Otsuka, Japan), the skin was closed using 4- 0 absorbable dermal sutures (4-0 Monodiox, Alfresa Pharma Corporation, Japan).

\section{Statistical analysis}

The frequency of the occurrence of postoperative complications (Clavien-Dindo Classification III or more) was compared using Fisher's exact test. $p<0.05$ was considered statistically significant. No other analysis or multivariate analysis was planned owing to the small number of included patients. For statistical analysis, EZR version 1.40 was used.

\section{Results}

\section{Patient characteristics}

The mean age of the patients was 66.1 years, and 59.6\% patients were male (Table 1). Stoma closure within 90 days (EC group) was performed in 29 (62\%) of 47 cases. Most patients had been diagnosed with early rectal cancer, and they were treated with laparoscopic lower anterior resection. Preoperative chemotherapy was provided only in 1 patient in the EC group. Adjuvant chemotherapy was performed in $19 \%$ of patients, mainly for Stage III disease.

The mean (median) interval time between initial surgery and stoma closure was 94.9 days [range; 15-371 days]. The mean time to stoma closure was 57.2 days (range: $15-90$ days) and 156.8 days (range: $91-371$ days) in the EC and LC groups, respectively. 
Table 1 Clinical features of the patients

\begin{tabular}{|c|c|c|c|}
\hline & $\begin{array}{l}\text { Total } \\
N=47\end{array}$ & $\begin{array}{l}\text { EC group } \\
N=29\end{array}$ & $\begin{array}{l}\text { LC group } \\
N=18\end{array}$ \\
\hline \multicolumn{4}{|l|}{ Age } \\
\hline year, Median [range] & 68 [33-86] & 69 [33-86] & 66.5 [48-79] \\
\hline \multicolumn{4}{|l|}{ Gender } \\
\hline Male/Female & $28 / 19$ & $15 / 14$ & $13 / 5$ \\
\hline \multicolumn{4}{|l|}{ Tumor location } \\
\hline $\mathrm{Rs} / \mathrm{Ra} / \mathrm{Rb}$ & $1 / 8 / 38$ & $1 / 7 / 21$ & $0 / 1 / 17$ \\
\hline \multicolumn{4}{|l|}{ Stage } \\
\hline । & $24(51.1)$ & $16(55.2)$ & $8(44.4)$ \\
\hline$\|$ & $8(17.0)$ & $4(13.8)$ & $4(22.2)$ \\
\hline III & $9(19.1)$ & $5(17.2)$ & $4(22.2)$ \\
\hline IV & $5(10.6)$ & $4(13.8)$ & $1(5.6)$ \\
\hline Other & $1(2.1)$ & 0 & $1(5.6)$ \\
\hline \multicolumn{4}{|l|}{ Approach and surgery } \\
\hline Lap-LAR & $38(80.9)$ & $22(75.9)$ & $16(88.9)$ \\
\hline Open-LAR & $4(8.5)$ & $4(13.8)$ & 0 \\
\hline Lap-LAR(Trans-anal) & $3(6.4)$ & $3(10.3)$ & 0 \\
\hline Open-LAR(Trans-anal) & $2(4.3)$ & 0 & $2(11.1)$ \\
\hline \multicolumn{4}{|l|}{ Adjuvant chemotherapy } \\
\hline Yes (\%) & $9(19.2)$ & $3(10.3)$ & $6(33.3)$ \\
\hline \multicolumn{4}{|c|}{ Neoadjuvant chemotherapy } \\
\hline Yes (\%) & $1(2.1)$ & $1(3.5)$ & $0(0)$ \\
\hline
\end{tabular}

Interval between creation and closure of stoma

day, Median [range] $\quad 79$ [15-371] 58 [15-90] 107.5 [91-371]

Lap- laparoscopic assisted, LAR low anterior resection, Trans-anal trans-anal anastomosis

\section{Postoperative complications after initial treatment}

Postoperative complications developed in 23 (49\%) of 47 patients; however, there were no surgery-related deaths (Table 2). Anastomotic leakage was observed in 4 patients (9\%). In EC group, $24 \%$ of the patients suffered from the complications related to the ileostomy, such as obstruction of the jejunum when it passed through the abdominal wall, internal hernia of oral-side small intestine related to the stoma, and ileus due to adhesion of the jejunum to the abdominal wall around the stoma. Meanwhile, $5.5 \%(1 / 18)$ of patients in the LC group developed these complications. Therefore, we found that stomarelated complications that were refractory to conservative treatment often resulted in early stoma closure (Table 2).

\section{Complications after stoma closure}

Overall, the rate of postoperative complications was $48.3 \%$ and $27.8 \%$ in the EC and LC groups, respectively (Table 3; Fig. 1). Within the EC group, the complications seemed equally distributed over 30 days (Fig. 1a). In terms of Clavien-Dindo Classification I/II complications, the rate was $27.6 \%$ and $11.2 \%$ in the $\mathrm{EC}$ and $\mathrm{LC}$ groups, respectively. We found that superficial incisional surgical site infections (SSI) developed in 5 and 1 patients in the EC and LC groups, respectively (Fig. 1b). However, the rate of Clavien-Dindo Classification III or higher complications was similar between the groups: $20.7 \%$ and $16.7 \%$ in the EC and LC groups, respectively ( $p=1$, Fisher's exact test) (Fig. 1c). These complications included colo-rectal/colo-anal anastomotic stenosis, urinary retention necessitating catheter placement, abscess, ileus, abdominal incisional hernia, and recto-vaginal fistula. Anastomotic leakage of the colorectal anastomosis was not observed. When only the complications within 30days after operation are counted, the occurrence rate of complications with Clavien-Dindo Classification III or higher is $13.7 \%(4 / 29)$ for EC group and 5\% (1/18) for LC group respectively, which has no statistical difference.

\section{Discussion}

This retrospective study showed no association between the time of closure and development of major complications. However, EC is likely associated with higher occurrence of minor complications. This study is uniquely valuable because of the heterogeneous characteristics of the patients, including a wide age range, the Eastern

Table 2 Postoperative complications occurring between initial operation and stoma closure

\begin{tabular}{|c|c|c|c|}
\hline & $N=47$ & EC group $N=29$ & LC group $N=18$ \\
\hline \multicolumn{4}{|c|}{ The number of patients with postoperative complications } \\
\hline & $23(48.9)$ & $14(48.3)$ & $9(50.0)$ \\
\hline \multicolumn{4}{|l|}{ Incidence of the complications } \\
\hline Anastomotic leakage & $4(8.5)$ & $2(6.9)$ & $2(11.1)$ \\
\hline Ileostomy-related ileus & $8(17.0)$ & $7(24.1)$ & $1(5.6)$ \\
\hline Other intestinal obstruction & $6(12.8)$ & $3(10.3)$ & $3(16.7)$ \\
\hline Abdominal abscess & 0 & 0 & 0 \\
\hline Other complications $^{a}$ & $5(10.6)$ & $2(6.9)$ & $3(16.7)$ \\
\hline
\end{tabular}

The number in the parenthesis is percentage

${ }^{a}$ Other complications included allergic reaction against drug used perioperative period, anemia without bleeding, anxiety after surgery, and recto-bladder fistula 
Table 3 Postoperative complications after stoma closure within 1 year

\begin{tabular}{|c|c|c|}
\hline & $\begin{array}{l}\text { EC group } \\
N=29\end{array}$ & LC group $N=18$ \\
\hline \multicolumn{3}{|l|}{ All complications } \\
\hline & $14(48.3)$ & $5(27.8)$ \\
\hline \multicolumn{3}{|l|}{ Calvien-Dindo classification } \\
\hline 1 & $4(13.8)$ & $1(5.6)$ \\
\hline 2 & $4(13.8)$ & $1(5.6)$ \\
\hline 3а & $4(13.8)$ & 0 \\
\hline $3 b$ & $2(6.9)$ & $3(16.7)$ \\
\hline Calvien-Dindo classification $\geq 3^{*}$ & $6(20.7)$ & $3(16.7)$ \\
\hline \multicolumn{3}{|l|}{ Type of complications } \\
\hline Anastomotic leakage (colo-anal) & 0 & 0 \\
\hline Abdominal abscess & $1(3.4)$ & 0 \\
\hline Wound infection of abdominal wall & $5(17.2)$ & $1(5.6)$ \\
\hline Others & $8(27.6)$ & $4(22.2)$ \\
\hline Anastomotic stenosis & $1(3.4)$ & $1(5.6)$ \\
\hline Recto-vaginal fistula & 0 & $1(5.6)$ \\
\hline Abdominal hernia & 0 & $1(5.6)$ \\
\hline Bowel obstruction & $2(6.9)$ & 0 \\
\hline Urinary retention & $1(3.4)$ & 0 \\
\hline Cellulitis at the drop site & $1(3.4)$ & 0 \\
\hline Prolonged fever & $2(6.9)$ & 0 \\
\hline Diarrhea & $1(3.4)$ & $1(5.6)$ \\
\hline
\end{tabular}

Statistical comparison was performed only to compare the frequency of complication with Calvien-Dindo classification $\geq 3 *$, because the number of the included patients are limited in the present study. The data analysis didn7t reveal the significant difference between two groups (Fisher's exact test, $P=1$ )

Cooperative Oncology Group Performance Status Scale (ECOG-PS), and postoperative complications after initial surgery $[12,13]$. Furthermore, the study demonstrated two intriguing results.

First, early stoma closure within 90 days after the initial operation was not associated with seriouscomplications such as leakage of the colorectal/coloanal anastomosis, or abdominal abscess formation. The total complication rate of Clavien-Dindo Classification III or more was $20.7 \%$ and $16.7 \%$ in the EC and LC groups (Table 3), respectively, suggesting no significant difference between the groups. Thus, we can assume that early stoma closure is a feasible management option when preoperative radiological examinations suggest no anastomotic leakage. However, one concern is that the frequency of severe postoperative complications (Clavien-Dindo Classification $\geq$ III) in the study is greater than that reported in previous studies [12, 13]. Danielsen reported a complication rate of $3.6 \%$ and $7 \%$ in the EC and LC groups, respectively [12]. Inclusion of old cases and a longer observation period after stoma closure may partially explain the high incidence of postoperative complications in our study. However, further improvement in the high rate of postoperative complications in our institute is needed.

The next clinical question to be answered is, what is the best timing for stoma closure within 90 days? A clinical trial demonstrated that stoma closure at 30 days after the initial operation increased the rate of severe postoperative complications when compared with that of late stoma closure [18]. Although the authors could not explain the reasons for their results, adhesion at 30 days after the initial operation could be worse than after a longer period, and difficulties during the operation might result in higher occurrence of severe postoperative complications. In the present study, only three patients underwent stoma reversal 20-40 days after the initial surgery. However, we should note that one of these patients developed a pelvic abscess after stoma closure. Further studies should focus on analyzing the safety of stoma closure approximately 1 month after the initial operation. We believe that elective stoma closures within 1 month should be avoided until clear evidence of their safety has been established.

The second intriguing point is the possibility of an increased incidence in minor postoperative complications, Clavien-Dindo Classification I/II, after early stoma closure. For instance, superficial incisional SSI was observed in $17.2 \%(5 / 29)$ and $5.6 \%(1 / 18)$ of patients after early and delayed closure, respectively (Table 3 ). One explanation for this could be that early stoma closure in our patients was performed when stoma-related complications could not be conservatively resolved rather than when the postoperative course was uneventful. Therefore, patients who underwent early stoma closure could have had a worse general condition, or local inflammation around the stoma, leading to the high incidence of minor postoperative complications. Our institution started to adopt a purse-string closure technique, which is associated with significantly fewer SSIs and better cosmetic outcomes with stoma reversal than those associated with conventional primary closure [19], thus potentially reducing the postoperative wound infection rate even after early stoma closure. Furthermore, applying anti-adhesive materials [20] around the stoma could reduce the technical difficulties experienced and further improve the results of early stoma closure.

The limitations of this study are the small number of patients and the unadjusted comparison between the two groups. Because the occurrence of postoperative complications is multi-factorial, a simple comparison may exaggerate or mask the differences. Thus, we focused on the statistical comparison of severe complications, resulting in the obscurity of other complications. Further, we could not assess the safety of "very" early stoma closure, within 2 weeks of the initial surgery. 

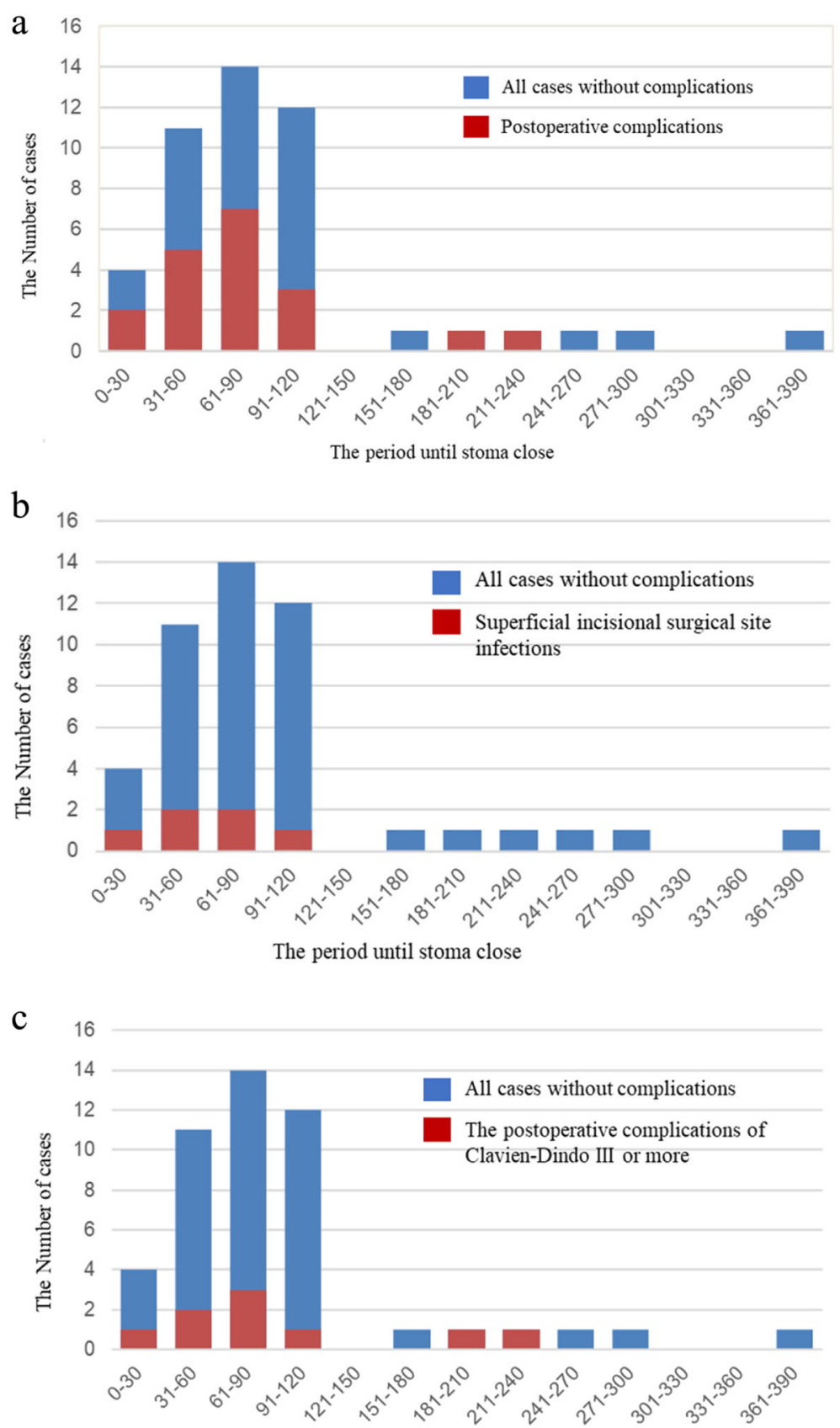

The period until stoma close

Fig. 1 The interval between stoma creation and closure and the occurrence of postoperative complications. Bar graphs show the number of stoma closures per period (30 days) after the initial operation. The number of postoperative complications among the cases is shown in red (a) All cases and postoperative complications are demonstrated. The postoperative complications accumulate within 90 days. In the early closure (EC) group, the complications seem to be equally distributed. $\mathbf{b}$ The accumulation of superficial incisional surgical site infections among patients who underwent stoma closure within 90 days. c Postoperative Clavien-Dindo Classification of III or higher complications are highlighted in red

Although clinical trials have suggested the safety of very early stoma closure in selected patients, further studies with the inclusion of older patients are necessary before recommending early stoma closure for all patients. Furthermore, a small number of patients who underwent preoperative chemotherapy (or chemo-radiation) were included in this study. Hence, our results cannot be extrapolated in these clinical settings because of the potential risks associated with early stoma closure due to the poor clinical condition of these patients [21].

We are currently conducting and awaiting the results of a clinical trial investigating whether very early stoma 
closure within 2 weeks of initial surgery is safe. (UMIN ID: 000036382, registered on 03/04/2019).

\section{Conclusions}

This study did not suggest that early stoma closure performed within 90 days after initial surgery increased the frequency of life-threatening, severe postoperative complications, even in vulnerable patients such as the elderly. However, the safety of early stoma reversal within 2 weeks after the initial operation, which is often discussed in the literature, was not answered from the present study. The results of this study can be used to develop clinical trials that reflect the outcomes of early stoma closure in the general population and may be used to develop future treatment guidelines.

\section{Abbreviations \\ EC: Early closure; LC: Late closure; SSI: Surgical site infection; ECOG- PS: Eastern Cooperative Oncology Group Performance Status Scale}

\section{Acknowledgements}

The authors thank the Kochi Organization for Medical Reformation and Renewal for their support in this research.

\section{Authors' contributions}

Hajime Kuroiwa analyzed and interpreted the patient data. Hiromichi Maeda was a major contributor in writing the manuscript. All authors read and approved the final manuscript.

\section{Funding}

No funding was received for this study.

\section{Availability of data and materials}

Not applicable.

\section{Ethics approval and consent to participate}

This study was approved by the Institutional Review Board for Clinical Research of the Kochi Medical School (ERB-103996, 30-44) and performed in accordance with the Declaration of Helsinki. An opt-out system was in place for participant consent.

\section{Consent for publication}

Not applicable.

\section{Competing interests}

The authors declare that they have no competing interests.

\section{Author details}

'Department of Surgery, Kochi Medical School, Kohasu, Oko-cho, 783-8505 Nankoku-city, Kochi, Japan. ${ }^{2}$ Cancer Treatment Center, Kochi Medical School, Kohasu, Oko-cho, Nankoku-city 783-8505, Kochi, Japan. ${ }^{3}$ Integrated Center for Advanced Medical Technologies (ICAM-Tech), Kochi Medical School, Nankoku-city, Japan.

Received: 19 October 2020 Accepted: 7 December 2020 Published online: 10 January 2021

\section{References}

1. Taflampas P, Christodoulakis M, Tsiftsis DD. Anastomotic leakage after low anterior resection for rectal cancer: facts, obscurity, and fiction. Surg Today 2009;39(3):183-8.

2. Snijders HS, Wouters MW, van Leersum NJ, Kolfschoten NE, Henneman D, de Vries AC, et al. Meta-analysis of the risk for anastomotic leakage, the postoperative mortality caused by leakage in relation to the overall postoperative mortality. Eur J Surg Oncol. 2012;38(11):1013-9.
3. Lee SY, Kim CH, Kim YJ, Kim HR. Impact of anal decompression on anastomotic leakage after low anterior resection for rectal cancer: a propensity score matching analysis. Langenbecks Arch Surg. 2015;400(7): $791-6$.

4. Matsuda M, Tsuruta M, Hasegawa H, Okabayashi K, Kondo T, Shimada T, et al. Transanal drainage tube placement to prevent anastomotic leakage following colorectal cancer surgery with double stapling reconstruction. Surg Today. 2016;46(5):613-20.

5. Tanaka K, Okuda J, Yamamoto S, Ito M, Sakamoto K, Kokuba Y, et al. Risk factors for anastomotic leakage after laparoscopic surgery with the double stapling technique for stage 0/I rectal carcinoma: a subgroup analysis of a multicenter, single-arm phase II trial. Surg Today. 2017;47(10):1215-22.

6. Matthiessen P, Hallböök O, Rutegård J, Simert G, Sjödahl R. Defunctioning stoma reduces symptomatic anastomotic leakage after low anterior resection of the rectum for cancer: a randomized multicenter trial. Ann Surg. 2007;246(2):207-14

7. Mrak K, Uranitsch S, Pedross F, Heuberger A, Klingler A, Jagoditsch M, et al. Diverting ileostomy versus no diversion after low anterior resection for rectal cancer: A prospective, randomized, multicenter trial. Surgery. 2016; 159(4):1129-39.

8. Kim YA, Lee GJ, Park SW, Lee WS, Baek JH. Multivariate Analysis of Risk Factors Associated With the Nonreversal Ileostomy Following SphincterPreserving Surgery for Rectal Cancer. Ann Coloproctol. 2015;31(3):98-102.

9. Abe S, Kawai K, Nozawa H, Hata K, Kiyomatsu T, Tanaka T, et al. Use of a nomogram to predict the closure rate of diverting ileostomy after low anterior resection: A retrospective cohort study. Int J Surg. 2017:47:83-8.

10. Herrle F, Sandra-Petrescu F, Weiss C, Post S, Runkel N, Kienle P. Quality of Life and Timing of Stoma Closure in Patients With Rectal Cancer Undergoing Low Anterior Resection With Diverting Stoma: A Multicenter Longitudinal Observational Study. Dis Colon Rectum. 2016;59(4):281-90.

11. O'Leary DP, Fide CJ, Foy C, Lucarotti ME. Quality of life after low anterior resection with total mesorectal excision and temporary loop ileostomy for rectal carcinoma. Br J Surg. 2001:88(9):1216-20.

12. Danielsen AK, Park J, Jansen JE, Bock D, Skullman S, Wedin A, Marinez AC, et al. Early closure of a temporary ileostomy in patients with rectal cancer: a multicenter randomized controlled trial. Ann Surg. 2017;265(2):284-90.

13. Alves A, Panis Y, Lelong B, Dousset B, Benoist S, Vicaut E. Randomized clinical trial of early versus delayed temporary stoma closure after proctectomy. Br J Surg. 2008;95(6):693-8.

14. Kotake K, Asano M, Ozawa H, Kobayashi H, Sugihara K. Gender differences in colorectal cancer survival in Japan. Int J Clin Oncol. 2016;21(1):194-203.

15. Matsuoka H, Maeda K, Hanai T, Sato H, Masumori K, Koide $Y$, et al. Surgical management of colorectal cancer for the aging population-A survey by the Japanese Society for Cancer of Colon and Rectum. Asian J Surg. 2018;41(2): 192-6.

16. Shiga M, Maeda H, Oba K, Okamoto K, Namikawa T, Fujisawa K, et al. Safety of laparoscopic surgery for colorectal cancer in patients over 80 years old: a propensity score matching study. Surg Today. 2017:47(8):951-8.

17. Lowry AC, Simmang CL, Boulos P, Farmer KC, Finan PJ, Hyman N, et al. Consensus statement of definitions for anorectal physiology and rectal cancer. Colorectal Dis. 2001;3(4):272-5.

18. Bausys A, Kuliavas J, Dulskas A, Kryzauskas M, Pauza K, Kilius A, et al. Early versus standard closure of temporary ileostomy in patients with rectal cancer: A randomized controlled trial. J Surg Oncol. 2019;120(2):294-9.

19. Hsieh MC, Kuo LT, Chi CC, Huang WS, Chin CC. Pursestring closure versus conventional primary closure following stoma reversal to reduce surgical site infection rate: a meta-analysis of randomized controlled trials. Dis Colon Rectum. 2015;58(8):808-15

20. Suto T, Watanabe M, Endo T, Komori K, Ohue M, Kanemitsu Y, et al. The Primary Result of Prospective Randomized Multicenter Trial of New SprayType Bio-absorbable Adhesion Barrier System (TCD-11091) Against Postoperative Adhesion Formation. J Gastrointest Surg. 2017;21(10):1683-91.

21. Yin TC, Tsai HL, Yang PF, Su WC, Ma CJ, Huang CW, et al. Early closure of defunctioning stoma increases complications related to stoma closure after concurrent chemoradiotherapy and low anterior resection in patients with rectal cancer. World J Surg Oncol. 2017;15(1):80.

\section{Publisher's Note}

Springer Nature remains neutral with regard to jurisdictional claims in published maps and institutional affiliations. 